\title{
9
}

\section{INSTRUMENTOS DEL INSTITUTO DE CRÉDITO OFICIAL PARA LA FINANCIACIÓN DE LA INTERNACIONALIZACIÓN DE LA EMPRESA ESPAÑOLA}

El ICO forma parte del Plan de Acción para la Internacionalización de la Economía Española 2019-2020. En el presente artículo se expone el importante papel que lleva a cabo el Instituto de Crédito Oficial en su función de banco público para la financiación de la actividad e inversiones empresariales tanto en España como en mercados internacionales, con el objetivo de promover el crecimiento económico y la generación de empleo. La financiación del ICO a las empresas se articula en diversas modalidades, recurriendo a esquemas de colaboración público-privada a través de sus diferentes instrumentos, que se pueden clasificar en tres áreas de actividad: Líneas ICO de Mediación; financiación directa mediante estructuraciones en distintas modalidades; y private equity o capital riesgo, en este último caso a través de su filial AXIS.

Palabras clave: internacionalización, exportación e inversión internacional, crecimiento empresarial, empresas españolas, pymes, entidades financieras, mercados exteriores, financiación internacional, mercados y sector financiero.

Clasificación JEL: F20, F30.

\section{Introducción: naturaleza y} actividades de ICO y contribución a la internacionalización de las empresas españolas

El Instituto de Crédito Oficial (ICO) está adscrito al Ministerio de Economía y Empresa a través de la Secretaría de Estado de Economía y Apoyo a la Empresa. ICO es una entidad de

\footnotetext{
* Presidente de ICO, Técnico Comercial y Economista del Estado, coordinador de este artículo junto con Elena Aranda, Directora de Mediación de Líneas ICO; Ana Martínez y Begoña Amores, Jefas de Área; y Mercedes Storch, Jefa de Relaciones Internacionales de AXIS.

Versión de junio de 2019.

DOI: https:/doi.org/10.32796/bice.2019.3114.6889
}

crédito pública, que actúa principalmente como banco público de promoción del crecimiento económico y agencia financiera del Estado. Para llevar a cabo esta actividad, ICO se financia en los mercados de capitales. Las deudas y obligaciones que contrae gozan frente a terceros de la garantía explícita, directa e irrevocable del Estado.

En primer lugar, como banco público de promoción del crecimiento económico contribuye -en colaboración y de forma complementaria con las entidades de crédito privadas- a dinamizar la financiación de todas las empresas, especialmente pymes y autónomos, para favorecer su crecimiento, actividades en general $D$ 
e inversiones tanto en España como en mercados exteriores. Para ello, ICO dispone de diferentes fórmulas de financiación, que son objeto de análisis en este artículo: Líneas ICO, financiación directa y vías complementarias al crédito bancario.

Estos esquemas permiten el acompañamiento a las empresas españolas en el desarrollo de sus actividades empresariales e inversiones, tanto en España como en el exterior, en etapas iniciales de internacionalización y en aquellas de expansión con fuerte presencia y vocación internacional.

Generalmente, la financiación ICO genera valor añadido en los plazos más largos y ofrece condiciones complementarias a los productos de las entidades financieras. En todas las operaciones, ICO trata de trasladar a las empresas las mejores condiciones posibles, en función de la situación de los mercados financieros y de capitales.

Para contextualizar la actividad que desarrolla ICO en el ámbito de la financiación empresarial, cabe señalar que el saldo vivo de crédito y avales otorgado por el banco público a empresas ascendía a 20.500 millones de euros a principios de 2019, focalizándose en España más del $80 \%$ de la financiación total concedida. El saldo vivo de crédito a la internacionalización prestado por ICO a las empresas se sitúa en torno a los 2.900 millones de euros.

Los recursos destinados por ICO a la internacionalización de las empresas españolas son crecientes y actualmente representan entre el $20 \%$ y el $30 \%$ del total de los flujos de la actividad crediticia, según el año. En los últimos tres años más de 5.500 empresas exportadoras han obtenido flujos crediticos de ICO por importe total de 4.557 millones de euros destinados a promover sus exportaciones de bienes o servicios a mercados de otros países o a inversiones en el exterior.

En segundo lugar, ICO actúa como instrumento financiero para desarrollar e implementar medidas de política económica del Gobierno.

En tercer lugar, ICO lleva a cabo, fuera de balance, servicios financieros como agencia financiera del Estado para gestionar, por cuenta de otros ministerios ${ }^{1}$, determinados instrumentos. En materia de internacionalización, cabe destacar que ICO actúa siempre por cuenta y bajo las indicaciones de la Secretaría de Estado de Comercio del Ministerio de Industria, Comercio y Turismo. En este marco, ICO gestiona el FIEM -Fondo de Internacionalización de la Empresa- y el CARI -Contrato de Ajuste Recíproco de Inversiones- conforme al consenso de la OCDE en materia de exportación. Los activos bajo gestión de ambos instrumentos superan un saldo vivo de más de 7.000 millones de euros y están movilizando de media más de 600 millones de euros anuales en casi veinte operaciones al año.

ICO focaliza esfuerzos para potenciar su papel en el ámbito internacional como instrumento financiero para apoyar medidas de política económica. En este marco destaca la iniciativa estratégica de posicionar a ICO como banco público de promoción acreditado ante la Unión Europea en el Marco Financiero Plurianual 2021-2027 a través del programa InvestEU. Esta acreditación constituye un paso más en la importante labor que el instituto ha venido desarrollando como catalizador de fondos de la Unión Europea para las empresas españolas.

Concretamente, ICO ha contribuido con 1.713 millones de euros de financiación total $\triangleright$

ICO actúa además como agente financiero del Estado por cuenta del MAEC y de la AECID en la gestión del FONPRODE y del Fondo del Agua, y por cuenta del Ministerio de Hacienda Pública en la gestión de los Fondos de Financiación a CC AA y CC LL. 
en proyectos apoyados por EFSI (European Fund for Strategic Investments), más conocido como Plan Juncker.

El impulso a la actividad internacional de las empresas españolas es una de las señas de identidad del Instituto de Crédito Oficial y parte fundamental de su estrategia. Las acciones que ICO lleva a cabo en este ámbito están alineadas con la Estrategia de Internacionalización de la Economía Española del Ministerio de Industria, Comercio y Turismo y en el último Plan de Acción para la Internacionalización de la economía Española 2019-2020.

Este artículo se centra en explicar el funcionamiento de los tres principales mecanismos de los que dispone ICO, con especial referencia en materia de internacionalización de las empresas. En el primer apartado se describen las Líneas ICO de Mediación, que se comercializan a través de la intermediación de los bancos y que otorga la capilaridad a ICO para llegar a todas las empresas. En el segundo apartado se exponen las estructuraciones en diferentes modalidades de financiación directa que ICO puede ofrecer a las empresas participando junto a los bancos de las mismas. En el tercer apartado, y de manera muy breve, algunas orientaciones generales sobre la financiación disponible mediante private equity o capital riesgo, en este último caso a través de su filial AXIS.

El análisis llevado a cabo integra las novedades que se han introducido para este año 2019 en los diferentes instrumentos de financiación, con base en la reorientación de la estrategia de ICO 2019-2021. Estas novedades se concretan en medidas y mejoras de las condiciones financieras de los productos que contribuyan al crecimiento de la actividad crediticia de ICO y se adapten a las necesidades del sector empresarial privado y faciliten la colaboración público-privada.

\section{Líneas ICO de Mediación}

Dentro del rol de ICO como banco público se enmarcan las denominadas Líneas ICO, cuya finalidad es otorgar financiación a empresas, emprendedores y autónomos a través de la intermediación de las entidades financieras privadas. Las Líneas ICO son uno de los pilares básicos de la actividad crediticia de ICO y un claro ejemplo de la eficiencia y eficacia de un modelo de colaboración público-privada.

Las Líneas ICO están destinadas a empresas de todos los tamaños - especialmente pymes- para apoyar financieramente el desarrollo de sus actividades e inversiones con el objetivo de favorecer la expansión y crecimiento empresarial, tanto en el ámbito nacional como internacional.

Las empresas tramitan las solicitudes de financiación ICO dentro de su circuito habitual bancario, acudiendo a su entidad para la financiación de su proyecto inversor o para el desarrollo de su actividad empresarial y sus necesidades de liquidez. Por su parte, las entidades financieras son las encargadas de realizar el análisis y viabilidad de los proyectos, concesión de la financiación, asumiendo el riesgo de las operaciones. La utilización de la red de oficinas de la práctica totalidad de las entidades financieras ha permitido la máxima optimización y capilaridad en la comercialización del crédito a todas las empresas.

Estos productos ofrecen largos plazos de amortización, amplias carencias, sin importe de financiación mínimo e importe máximo de 12,5 millones de euros y tipos de interés, que se encuentran publicados en la web $\mathrm{ICO}^{2}$, y que se establecen como tipos máximos de la entidad a la empresa, por lo que, en condiciones competitivas la empresa puede obtener $\triangleright$

2 Web ICO: www.ico.es 
tipos más bajos en su financiación ICO (por ejemplo, financiaciones ICO a 5 años están obteniendo en media un tipo nominal del 2,13\%). Además, las líneas aportan financiación en cualquier zona geográfica y para cualquier sector de actividad, tanto en euros como en dólares.

Las Líneas ICO ofrecen un amplio catálogo de productos financieros, agrupadas en dos grandes áreas de actividad: Líneas ICO Nacionales para la financiación a empresas, emprendedores y autónomos, cuya actividad empresarial y proyectos de inversión se lleven a cabo en España, y Líneas ICO Internacionales para la financiación de empresas, de cualquier tamaño, en su actividad internacional y exportadora.

\subsection{Líneas ICO Nacionales}

Su finalidad es financiar a las empresas para llevar a cabo sus actividades empresariales y/o inversiones, atender sus necesidades de liquidez y gastos relacionados con el desarrollo de la actividad empresarial dentro del territorio nacional.

Para ello, las líneas están disponibles para cualquier sector de actividad y para cualquier empresa sin limitación de tamaño. Son productos muy capilares y accesibles, con un importe medio de financiación en torno a los 70.000 euros, considerando que el importe máximo es de hasta 12,5 millones de euros por empresa.
Además, las Líneas ICO aportan especialización ofreciendo largos plazos de amortización de hasta 20 años, amplias carencias del capital de hasta 3 años, tipos de interés competitivos y proceso de solicitud y tramitación de la operación simple y accesible, a través de la propia entidad financiera de la empresa.

El catálogo integral de Líneas ICO Nacionales está formado por los siguientes productos:

- ICO Empresas y Emprendedores, para la financiación de toda la actividad empresarial que lleve a cabo la empresa en territorio nacional, tanto para inversión como para liquidez y gastos de la actividad habitual.

- ICO Crédito Comercial, para la financiación, como línea de liquidez revolving a corto plazo, de los anticipos de facturas y prefinanciación de la actividad comercial, dentro de territorio nacional, llevada a cabo por autónomos y empresas.

- ICO Garantía SGR/SAECA, para la financiación de toda la actividad empresarial que lleve a cabo la empresa en territorio nacional (también puede tener garantía SGR si el proyecto se lleva fuera de España), tanto para inversión como para liquidez y gastos de la actividad habitual, con el valor añadido de la garantía aportada por una Sociedad de Garantía Recíproca.

CUADRO 1

CATÁLOGO DE LÍNEAS ICO NACIONALES

\begin{tabular}{|l|l|}
\hline $\begin{array}{l}\text { ICO Empresas y } \\
\text { Emprendedores }\end{array}$ & $\begin{array}{l}\text { Financiación de las actividades empresariales y/o inversiones que las empresas, emprendedores y } \\
\text { autónomos lleven a cabo dentro de España, así como sus necesidades de liquidez y gastos } \\
\text { relacionados con el desarrollo de la actividad propia de la empresa }\end{array}$ \\
\hline ICO Crédito Comercial & $\begin{array}{l}\text { Línea de liquidez revolving a corto plazo, que permite el anticipo de facturas y prefinanciación de la } \\
\text { actividad comercial llevada a cabo por autónomos y empresas en España }\end{array}$ \\
\hline ICO Garantía SGR/ SAECA & $\begin{array}{l}\text { Financiación de las actividades empresariales y/o inversiones que las empresas lleven a cabo tanto } \\
\text { dentro como fuera de territorio nacional, así como sus necesidades de liquidez, con la garantía de } \\
\text { una SGR }\end{array}$ \\
\hline Fuente: ICO. & \multicolumn{2}{|l}{}
\end{tabular}


En el Cuadro 1 se sintetizan las tres grandes líneas de financiación destinadas al apoyo financiero de la actividad nacional de nuestras empresas.

\subsection{Líneas ICO Internacionales}

ICO dispone de un catálogo integral de productos financieros adaptado a las necesidades y al perfil de las compañías con el objetivo de mejorar el acceso a los mercados exteriores por parte de las pymes. Este catálogo es ambicioso en cuanto a diseño de instrumentos de financiación exterior y se alinea con las mejores prácticas de financiación que desarrollan las instituciones financieras públicas internacionales especializadas en comercio internacional.

Tanto por el potencial de empresas como por definición de los productos y adecuación de las condiciones financieras - largos plazos de hasta 20 años, carencias de hasta 3 años, financiación en euro/dólar y en proyectos determinados en moneda local, flexibilidad en tramitación-, los productos de ICO contribuyen a facilitar las necesidades de financiación de las empresas españolas que abordan su salida al exterior:

- ICO Exportadores, para la financiación, como línea de liquidez revolving a corto plazo, de la actividad exportadora de la empresa, especialmente pyme, mediante el anticipo de facturas de la venta de bienes y/o servicios en el exterior, o la prefinanciación de su actividad anterior a la exportación.

- ICO Internacional, para la financiación de la actividad empresarial e inversora de la empresa con interés español en el exterior, con financiación a largo plazo en todas las etapas de su actividad. Además, en esta línea se financia la actividad exportadora a medio y largo plazo de la empresa, a través de la figura de crédito comprador y crédito suministrador y por importes de hasta 25 millones de euros.

- ICO Canal Internacional, para pymes y grandes empresas. En este producto es determinante la colaboración de entidades financieras internacionales, públicas y privadas, que actúan como mediadoras de la financiación ICO. Este esquema permite aportar recursos financieros a proyectos de inversión que tengan, directa o indirectamente, un componente de interés español en su desarrollo a largo plazo y en la financiación multidivisa -en la moneda local de los países en los que se desarrolla el proyecto-. Su objetivo es promover el papel de ICO como banco de segundo y tercer piso, en colaboración con las entidades financieras internacionales, que aportan $\triangleright$

CUADRO 2

CATÁLOGO DE LÍNEAS ICO INTERNACIONALES

\begin{tabular}{|l|l|}
\hline ICO Exportadores & Financiación a corto plazo de la actividad exportadora de la empresa \\
\hline ICO Internacional & Financiación de las actividades e inversiones empresariales que lleven a cabo fuera del territorio \\
\hline ICO Garantías Internacionales & $\begin{array}{l}\text { Garantías para operaciones de contratación internacional, tanto licitaciones como adjudicaciones } \\
\text { directas, públicas o privadas, ante entidades financieras privadas para promover el acceso a las } \\
\text { empresas españolas a mercados internacionales }\end{array}$ \\
\hline ICO Canal Internacional & $\begin{array}{l}\text { Financiación para la internacionalización de las empresas con interés español con la colaboración } \\
\text { de entidades financieras internacionales que actúan en mercado local }\end{array}$ \\
\hline Fuente: ICO & \\
\hline
\end{tabular}


como factor diferenciador el conocimiento del mercado y proximidad al cliente (KYC, know your coustomer).

- ICO Garantía Internacional, línea de avales y garantías internacionales ante entidades financieras que permite a las compañías españolas - públicas y privadas - participar en licitaciones y concursos internacionales, en condiciones competitivas.

En el Cuadro 2 se sintetizan las cuatro grandes líneas de financiación destinadas al apoyo financiero de la actividad internacional y exportadora de las empresas españolas.

\section{Crédito ICO a exportadores a corto plazo}

El comportamiento del sector exterior español ha resultado muy positivo en los últimos años, con un crecimiento elevado y sostenido de las exportaciones que ha permitido y contribuido a lograr un superávit de cuenta corriente en los años de recuperación económica. Esta afirmación viene avalada por las cifras que arrojan las exportaciones en los últimos años, respecto a la actividad económica global, alcanzando en 2017 su nivel más alto respecto al PIB con un $34 \%$, frente al $25 \%$ de hace diez años.

El peso de la actividad exportadora en las líneas respecto a la actividad global de financiación de ICO supone en 2018 un total del
$35 \%$, lo que refleja un comportamiento muy similar respecto a la evolución de las exportaciones del sistema y su peso respecto al total de la economía.

En el Cuadro 3 se refleja el peso de la actividad exportadora financiada a través de ICO sobre la financiación global ICO otorgada a empresas.

El perfil de empresa exportadora, que pone de manifiesto la capilaridad que tienen las Líneas ICO, responde en su mayor parte al perfil pyme, especialmente micro y pequeña empresa, con más del $52 \%$ total de financiación concedida. Las pymes se consideran el perfil de empresa con más potencial de crecimiento y la internacionalización les permite mejorar su competitividad y hacerse un hueco en el mercado global.

Si analizamos cómo varían el número total de empresas exportadoras, y de ellas cuáles son exportadoras recurrentes, vemos que el mayor impacto de crecimiento recae en las empresas pequeñas, que son las que se vienen incorporando al mercado exportador. Según datos del ICEX, el porcentaje de empresas que exportaron con regularidad en 2017 fue de 31 respecto al total de empresas exportadoras en ese año.

Al comparar este comportamiento con los datos de empresas exportadoras financiadas a través de la Línea ICO, cuya financiación habitualmente es complementaria de la $D$

CUADRO 3

PESO DE LA ACTIVIDAD DE EXPORTADORES SOBRE EL TOTAL DE LA FINANCIACIÓN DE LÍNEAS ICO (2016-2018)

(En millones de euros)

\begin{tabular}{|l|c|c|c|}
\hline & Actividad total & Actividad exportadores & \% sobre total \\
\hline 2016 & 4.681 & 1.196 & 26 \\
2017 & 4.515 & 571 & 13 \\
2018 & 2.337 & 820 & 35 \\
\hline Total & $\mathbf{1 1 . 5 3 3}$ & $\mathbf{2 . 5 8 7}$ & $\mathbf{2 2}$ \\
\hline Fuente: ICO.
\end{tabular}


financiación privada, observamos que en los años 2017 y 2018 el porcentaje de empresas regularmente exportadoras frente al total de empresas financiadas se sitúa por encima de 75 , lo cual es significativo dado el perfil de empresa financiado (Cuadro 4).

En lo que se refiere a la distribución de la financiación exportadora por sector de actividad, el sector industria tiene un peso de más del $57 \%$ sobre el total financiado en el periodo 2016-2018, seguido del sector del comercio, con un porcentaje superior a 26 y otros sectores destacados con porcentajes inferiores (Cuadro 5).

Por último, atendiendo a los principales destinos de las exportaciones de las empresas españolas que han recibido financiación a través de la Línea ICO, podemos concluir que geográficamente no existe una región o zona de mayor afluencia de exportación, destacando Francia como país vecino y primero en el ranking de destino, con una dispersión importante en mercados de otros países europeos y EE UU, entre otros (Cuadro 6).

\section{Crédito ICO a exportadores e inversiones en el exterior a medio y largo plazo}

Como se ha venido afirmando, el ICO, como entidad de crédito especializada, ha contribuido a la dinamización de la financiación a todas las empresas con especial foco en su salida al exterior. Concretamente, en los tres últimos años se han concedido a través de las Líneas ICO Internacional más de 313 millones de euros distribuidos en más de mil operaciones de empresas de distintos sectores de actividad y destinos (Cuadro 7).

CUADRO 4

DISTRIBUCIÓN DE LA FINANCIACIÓN DE LÍNEA ICO A EXPORTADORES (2016-2018): EXPORTADORES REGULARES

\begin{tabular}{|l|c|c|c|}
\hline Periodo & $\mathbf{N}^{\circ}$ de clientes & $\mathbf{N} .^{\circ}$ de clientes regulares & Total operaciones \\
\hline 2016 & 2.061 & 1.634 & 19.900 \\
2017 & 1.361 & 1.075 & 13.165 \\
2018 & 1.223 & 946 & 11.305 \\
\hline Fuente: ICO.
\end{tabular}

CUADRO 5

DISTRIBUCIÓN SECTORIAL DE LÍNEA ICO A EXPORTADORES (2016-2018)

\begin{tabular}{|c|c|c|c|c|}
\hline Sectores & Importe en mill. de $€$ & $\%$ sobre el total & Operaciones & $\%$ sobre el total \\
\hline Industria & $1.499,84$ & 57,99 & 23.133 & 52,14 \\
\hline Comercio & 683,44 & 26,42 & 12.390 & 27,92 \\
\hline Infraestructuras y transporte & 185,19 & 7,16 & 4.456 & 10,04 \\
\hline Actividades profesionales, consultoría y servicios & 71,20 & 2,75 & 1.369 & 3,09 \\
\hline Agricultura, ganadería y pesca & 64,24 & 2,48 & 1.470 & 3,31 \\
\hline Hostelería & 42,91 & 1,66 & 412 & 0,93 \\
\hline Comunicaciones y actividades artísticas & 26,11 & 1,01 & 740 & 1,67 \\
\hline Energía & 6,47 & 0,25 & 129 & 0,29 \\
\hline Otros servicios & 4,15 & 0,16 & 75 & 0,17 \\
\hline Sanidad y servicios sociales & 2,08 & 0,08 & 190 & 0,43 \\
\hline Educación & 0,77 & 0,03 & 6 & 0,01 \\
\hline Total & $2.586,40$ & 100,00 & 44.370 & 100,00 \\
\hline
\end{tabular}


José Carlos García de Quevedo Ruiz

CUADRO 6

DISTRIBUCIÓN POR PAÍSES DE LÍNEA ICO A EXPORTADORES (2016-2018)

\begin{tabular}{|l|c|c|c|c|}
\hline \multicolumn{1}{|c|}{ Países } & Importe en mill. de $\boldsymbol{\ell}$ & \% sobre el total & Operaciones & \% sobre el total \\
\hline Francia & 458,84 & 17,74 & 9.973 & 22,48 \\
Alemania & 252,46 & 9,76 & 3.667 & 8,26 \\
Italia & 249,51 & 9,65 & 3.941 & 8,88 \\
Portugal & 179,42 & 6,94 & 4.286 & 9,66 \\
Reino Unido & 175,98 & 6,80 & 3.044 & 6,86 \\
Estados Unidos & 128,24 & 4,96 & 2.094 & 4,72 \\
China & 98,92 & 3,82 & 571 & 1,29 \\
Países Bajos & 80,72 & 3,12 & 1.491 & 3,36 \\
Marruecos & 61,49 & 2,38 & 844 & 1,90 \\
México & 51,71 & 2,00 & 740 & 1,67 \\
Bélgica & 49,00 & 1,89 & 983 & 2,22 \\
Cuba & 45,07 & 1,74 & 383 & 0,86 \\
Suiza & 45,07 & 1,74 & 786 & 1,77 \\
Polonia & 36,39 & 1,41 & 568 & 1,28 \\
Arabia Saudí & 36,25 & 1,40 & 405 & 0,91 \\
Resto de países & 637,31 & 24,64 & 10.594 & 23,88 \\
\hline Total & $\mathbf{2 . 5 8 6 , 4 0}$ & $\mathbf{1 0 0 , 0 0}$ & $\mathbf{4 4 . 3 7 0}$ & $\mathbf{1 0 0 , 0 0}$ \\
\hline Fuente: ICo. & & & & \\
\hline
\end{tabular}

CUADRO 7

DISTRIBUCIÓN SECTORIAL DE LÍNEA ICO INTERNACIONAL (2016-2018)

\begin{tabular}{|c|c|c|c|c|}
\hline Sectores & Importe en mill. de $€$ & $\%$ sobre el total & Operaciones & $\%$ sobre el total \\
\hline Industria & 120,43 & 38,44 & 295 & 29,47 \\
\hline Comercio & 58,64 & 18,71 & 310 & 30,97 \\
\hline Infraestructuras y transporte & 46,99 & 15,00 & 135 & 13,49 \\
\hline $\begin{array}{l}\text { Actividades financieras, profesionales, científicas } \\
\text { y Administración Pública }\end{array}$ & 29,93 & 9,55 & 113 & 11,29 \\
\hline Agricultura, ganadería y pesca & 17,53 & 5,59 & 38 & 3,80 \\
\hline Otros servicios & 15,70 & 5,01 & 18 & 1,80 \\
\hline Comunicaciones y actividades artísticas & 13,78 & 4,40 & 65 & 6,49 \\
\hline Energía & 5,56 & 1,77 & 7 & 0,70 \\
\hline Educación & 3,17 & 1,01 & 8 & 0,80 \\
\hline Hostelería & 0,98 & 0,31 & 8 & 0,80 \\
\hline Sanidad y servicios sociales & 0,64 & 0,20 & 4 & 0,40 \\
\hline Total & 313,34 & 100,00 & 1.001 & 100,00 \\
\hline
\end{tabular}

La banca internacional en terceros países como nuevo socio: Línea ICO Canal Internacional al servicio de las empresas españolas

El contexto económico actual, con abundancia de liquidez en los mercados y tipos de interés bajos, supone un gran reto para el ICO, debiendo orientar su actividad hacia modelos de especialización, aportando valor añadido en aspectos como la financiación a largo plazo y el apoyo a la expansión internacional a otros mercados, y actuando sobre el terreno acompañando a las empresas españolas. 
INSTRUMENTOS DEL INSTITUTO DE CRÉDITO OFICIAL PARA LA FINANCIACIÓN...

CUADRO 8

DISTRIBUCIÓN POR PAÍSES DE LÍNEA ICO INTERNACIONAL (2016-2018)

\begin{tabular}{|c|c|c|c|c|}
\hline Países & Importe en mill. de $€$ & $\%$ sobre el total & Operaciones & $\%$ sobre el total \\
\hline Francia & 53,81 & 17,17 & 209 & 20,88 \\
\hline Italia & 31,01 & 9,90 & 73 & 7,29 \\
\hline Portugal & 26,62 & 8,50 & 79 & 7,89 \\
\hline Reino Unido & 20,68 & 6,60 & 49 & 4,90 \\
\hline México & 19,88 & 6,34 & 62 & 6,19 \\
\hline Alemania & 19,79 & 6,32 & 73 & 7,29 \\
\hline Estados Unidos & 15,25 & 4,87 & 51 & 5,09 \\
\hline R. D. Congo & 15,03 & 4,80 & 64 & 6,39 \\
\hline China & 14,38 & 4,59 & 42 & 4,20 \\
\hline Seychelles & 10,00 & 3,19 & 1 & 0,10 \\
\hline Chile & 8,73 & 2,79 & 19 & 1,90 \\
\hline Colombia & 7,17 & 2,29 & 19 & 1,90 \\
\hline Brasil & 6,53 & 2,08 & 15 & 1,50 \\
\hline Marruecos & 6,13 & 1,96 & 20 & 2,00 \\
\hline India & 5,20 & 1,66 & 10 & 1,00 \\
\hline Resto Países & 53,12 & 16,95 & 215 & 21,48 \\
\hline Total & 313,34 & 100,00 & 1.001 & 100,00 \\
\hline
\end{tabular}

Por ello, el ICO, en su vocación de apoyo internacional a la empresa española, promueve su papel como banco de segundo y tercer piso, en colaboración con las entidades financieras internacionales, tanto públicas como privadas, que aportan como factor diferenciador el conocimiento del mercado y proximidad al cliente (KYC) a través de la Línea ICO Canal Internacional. Para ello, ICO llega a acuerdos con bancos regionales o multilaterales, bancos de terceros países o filiales de bancos españoles en el exterior para financiar proyectos de interés español y para las empresas españolas o sus clientes.

Esta línea se caracteriza por su versatilidad, que permite atender las diferentes necesidades de la empresa española en su salida al exterior, con largos plazos de amortización y ofreciendo e impulsando la financiación multidivisa, aportando de esta forma recursos financieros a proyectos de inversión con interés español en la moneda local de los países en los que se desarrolla el proyecto.
Canal Internacional es una línea con fuertes perspectivas de crecimiento, habiendo suscrito, hasta la fecha, dieciséis acuerdos de financiación con trece contrapartidas internacionales, que permiten un alcance de financiación que cubre totalmente la actividad internacional, inversora y exportadora, de las empresas y proyectos con interés español en EE UU, Centroamérica y países LATAM, movilizando actualmente más de 1.500 millones de dólares.

Concretamente, se han firmado acuerdos con entidades multilaterales que operan en gran parte de la región: CAF (Banco de Desarrollo de América Latina), BCIE (Banco Centroamericano de Integración Económica) y Fonplata (Fondo Financiero para el Desarrollo de los Países de la Cuenca del Plata). En Estados Unidos, con Sadadell Miami; en México, con Bancomext, Sabadell México y BBVA Bancomer; en Colombia, con Bancóldex; en Perú, con Banbif, Cofide y BBVA Continental; en Argentina, con BICE. Actualmente se está $D$ 
negociando con otras entidades como BNDES para ampliar la cobertura del Canal Internacional lo máximo posible.

\section{Financiación directa}

El Instituto de Crédito Oficial pone a disposición de las empresas españolas financiación directa para el desarrollo de grandes proyectos de inversión tanto en España con en terceros mercados. La actividad de financiación directa de ICO responde a sus fines de sostenimiento $y$ promoción de actividades económicas que contribuyan al crecimiento y a la mejora de la distribución de la riqueza nacional y, en especial, de aquellas que por su trascendencia social, cultural, innovadora o ecológica merecen su fomento.

Adicionalmente, la actuación de ICO debe recoger su compromiso con un desarrollo económico sostenible tanto a nivel nacional como internacional, adecuándose a los estándares del mercado en cuanto a objetivos de sostenibilidad y cambio climático.

Las operaciones de financiación directa se agrupan -orientativamente pero no con carácter limitativo- en estos productos, que por su versatilidad se adaptan a las necesidades empresariales para favorecer su crecimiento $y$ proyección internacional:

- Créditos y préstamos corporativos.

- Préstamos sindicados.

- Club Deal.

- Project Finance o Financiación de Proyecto.

- Bonos corporativos.

- Avales financieros.

ICO puede ofrecer financiación tanto en el ámbito doméstico como en el internacional. La financiación deberá estar dirigida, independientemente de la estructura de la operación y de su instrumentación, a la realización de proyectos de inversión o innovación, desarrollo de actividades empresariales, exportación o planes corporativos de expansión en terceros mercados o en España.

ICO actúa siempre en colaboración y de forma complementaria con otras entidades financieras privadas o públicas nacionales, internacionales o multinacionales o regionales. Como norma general, la financiación ICO se sitúa en 10 millones de euros o su contravalor en otra divisa - excepcionalmente se podrá considerar un importe inferior - y no supera el mayor ticket de cualquier otra entidad.

El objetivo de la participación de ICO en estas operaciones es aportar valor añadido, especialmente en los tramos de financiación a más largo plazo. Además, la presencia de ICO posibilita en proyectos internacionales financiación en moneda local en países de difícil acceso, lo que facilita el cierre de las operaciones. En este sentido, cabe señalar que ICO ha sido pionero en la financiación externa en moneda local para proyectos internacionales de inversiones a largo plazo, contribuyendo a estructuras de colaboración público-privada con la banca de promoción local e intermediarios financieros privados.

El contexto económico de consolidación del crecimiento ha contribuido a la reactivación de la financiación directa para favorecer la expansión de medianas y grandes empresas y el desarrollo de proyectos de inversión. En 2018 se han formalizado operaciones directas de préstamo y aval por 1.190 millones de euros, de los que 724 millones han sido destinados a proyectos en el exterior, lo que representa más del $60 \%$. En términos de volúmenes dispuestos, a través de esta modalidad se han desembolsado 2.144 millones de euros (Cuadro 9). $D$ 
CUADRO 9

PRÉSTAMOS DIRECTOS Y AVALES DEL EJERCICIO 2018 DISTRIBUCIÓN GEOGRÁFICA POR DESTINO DE LA INVERSIÓN (En millones de euros)

\begin{tabular}{|l|c|c|}
\hline \multicolumn{1}{|c|}{ Tipo de inversión } & $\begin{array}{c}\text { Importe } \\
\text { formalizado }\end{array}$ & $\begin{array}{c}\text { Importe } \\
\text { dispuesto }\end{array}$ \\
\hline Inversión nacional & 466 & 1.443 \\
Inversión Internacional & 724 & 701 \\
\hline Total & $\mathbf{1 . 1 9 0}$ & $\mathbf{2 . 1 4 4}$ \\
\hline Fuente: ICO. & & \\
\hline
\end{tabular}

Sin duda, la financiación directa de proyectos internacionales adquiere especial relevancia en la actividad de ICO, en particular en los últimos años. ICO mantenía a finales de 2018 un saldo en proyectos internacionales con participación de empresas españolas financiados de forma directa equivalente a 1.600 millones de euros, distribuidos en trece países (Reino Unido, Portugal, Irlanda, Eslovaquia, Grecia, México, Chile, Perú, Colombia, Canadá, Australia, Emiratos Árabes y Arabia Saudí).

En la distribución de la cartera por sectores destacan las infraestructuras de transporte, la industria y la energía (Cuadro 10).

CUADRO 10 DISTRIBUCIÓN DE CARTERA POR SECTORES

\begin{tabular}{|l|c|c|}
\hline \multicolumn{1}{|c|}{$\begin{array}{c}\text { Financiación directa } \\
\text { internacional }\end{array}$} & $\begin{array}{c}\text { Saldo } \\
\text { vivo }\end{array}$ & $\begin{array}{c}\text { \% sobre } \\
\text { el total }\end{array}$ \\
\hline Infraestructuras y transporte & 524 & 32,2 \\
Industria & 465 & 28,6 \\
Energía & 429 & 26,4 \\
Servicios profesionales & 187 & 11,5 \\
Comercio & 16 & 1,0 \\
Hostelería & 6 & 0,4 \\
\hline Total & $\mathbf{1 . 6 2 9}$ & $\mathbf{1 0 0 , 0}$ \\
\hline Fuente: ICO. & & \\
\hline
\end{tabular}

\section{Fuentes de financiación alternativas. AXIS}

El apoyo financiero a las empresas no debe circunscribirse únicamente al crédito bancario.
Es necesario dinamizar otras alternativas, en particular las que puede ofrecer el capital riesgo. ICO, a través de su filial AXIS, invierte tanto en empresas como en fondos con el objetivo de promover el crecimiento de las empresas a largo plazo.

AXIS gestiona en la actualidad 2.650 millones de euros mediante los tres fondos que se exponen a continuación:

1. FOND-ICOpyme cuenta con unos recursos máximos disponibles de 250 millones de euros. Es un fondo generalista y su objetivo está enfocado principalmente a empresas que, habiendo alcanzado un cierto grado de madurez, quieran financiar su proceso de expansión, desarrollo tecnológico, crecimiento y/o internacionalización. Este fondo emplea sus recursos de dos formas distintas para lograr una mayor eficacia. Por un lado, utiliza la inversión directa para favorecer el crecimiento a largo plazo de las compañías líderes en su segmento de mercado, con criterios financieros, sin sustituir al emprendedor o equipo directivo y principalmente en régimen de coinversión. Por otro lado, tiene presencia de forma indirecta a través de los fondos en los que FOND-ICOpyme tiene participación, con objeto de lograr una mayor capilaridad en la distribución de sus recursos.

Desde su puesta en marcha, el fondo ha participado en forma de capital y préstamos participativos en más de sesenta compañías y en más de veinte fondos por un importe superior a los 220 millones de euros. Además, AXIS, a través de FOND-ICOpyme, ha colaborado en la puesta en marcha de diferentes iniciativas de financiación alternativa, como $\triangleright$ 
el lanzamiento junto al Fondo Europeo de Inversiones del European Business Angels Fund Spain. Se trata de un proyecto paneuropeo que persigue apoyar a los emprendedores españoles a través de la participación con inversores profesionales o business angels en las fases más iniciales de sus proyectos, favoreciendo su proyección exterior.

También destaca la iniciativa de Sostenibilidad e Impacto Social financiada por este fondo. Esta iniciativa se enmarca en la alineación que el instituto busca con los Objetivos de Desarrollo Sostenible y el cumplimiento de la Agenda 2030.

2. Fond-ICO Infraestructuras II cuenta con una dotación de 400 millones de euros y su objetivo principal es tanto la inversión directa en proyectos de infraestructura sostenible como la inversión a través de otros fondos de inversión en España y en el exterior con empresas españolas.

Las inversiones de este fondo se focalizan principalmente en infraestructuras de transporte, de energía y medio ambiente e infraestructura social.

En el segmento de infraestructuras, AXIS ha analizado en los últimos ocho años más de doscientos proyectos, de los cuales el $40 \%$ eran internacionales, en su mayoría del continente americano. En estos años, se han aprobado dos operaciones exteriores, en EE UU y en Chile, respectivamente. Recientemente, AXIS ha firmado su participación a través del fondo Fond-ICO Infraestructuras II en Talasol, proyecto para construir una planta fotovoltaica con una capacidad máxima de $300 \mathrm{MW}$ en Cáceres. Será una de las mayores plantas fotovoltaicas de España y contribuirá a la lucha contra el cambio climático. Esta operación cuenta con un préstamo del EFSI (European Fund for Strategic Investments) que pone de manifiesto la importancia de ICO/AXIS como cogenerador de proyectos de gran valor añadido e interés europeo, como ya se ha señalado en este artículo.

3. Fondo-ICO Global, con una dotación de 2.000 millones de euros, tiene por objeto promover la financiación de las pymes españolas a través de la inversión en fondos de capital privado con presencia en España. El principal objetivo de este fondo de fondos es impulsar la financiación de proyectos que combinen innovación y emprendimiento, invirtiendo en compañías que se encuentren en cualquiera de las fases de su desarrollo para que sean más competitivas.

Esta iniciativa ha demostrado una gran eficiencia durante los seis años de vigencia del programa, logrando un doble objetivo. En primer lugar, dinamizar el mercado de capital riesgo inyectando un volumen de recursos considerable. Así, a 31 de mayo de 2019, se han aprobado inversiones por un importe de 1.734 millones de euros en 82 fondos, de los cuales 1.077 millones de euros ya han sido suscritos y 580 millones de euros, desembolsados. En segundo lugar, y no por ello menos importante, la capilaridad de esta iniciativa ha permitido apoyar a 424 empresas españolas y promover la creación y el mantenimiento de más de 132.000 empleos.

\section{Conclusiones}

ICO trabaja con el objetivo de aportar valor añadido a la financiación de empresas de $\triangleright$ 
todos los tamaños y sectores para impulsar el crecimiento económico sostenible en línea con las políticas públicas recogidas a nivel internacional en la Agenda 2030 y reflejadas a nivel nacional en la Agenda del Cambio.

En este contexto, el apoyo a la actividad de las empresas españolas en terceros mercados adquiere especial relevancia, puesto que contribuyen al desarrollo de las regiones donde se implantan. Citemos, por ejemplo, el caso de Latinoamérica, donde las empresas españolas tienen una clara vocación de permanencia y contribución al desarrollo de la región, con un stock de inversión superior a los 120.000 millones de euros y casi 800.000 puestos de trabajo.

ICO presta especial atención a la promoción de la internacionalización de las empresas españolas en el marco de sus ejes estratégicos. Estos se sustentan en unos principios de actuación de carácter transversal en conexión con las prioridades de la Unión Europea -recogidas en el programa InvestEU- que se traducen en el desarrollo de la digitalización e innovación, impulso de la sostenibilidad y de la economía circular, y la promoción de fuentes de financiación alternativas para favorecer el crecimiento y la competitividad de las pymes, y los proyectos de inversión social y capacidades.

Como banco nacional de promoción, ICO seguirá adaptando la oferta de productos a las necesidades de las empresas para acompañarlas en su expansión en terceros mercados, intentando ofrecer un enfoque 360 grados en las opciones de financiación que plantea.

\section{Bibliografía}

AXIS (2017). Memoria de Sostenibilidad 2017. Recuperado de https://www.axispart.com/documents/1459996/1508286/1.2.1+Memoria+de+ Sostinibilidad+de+2017_AXIS.PDF/b8b17d6096ac-42cc-9c39-99e5d706c1ea

Cámara España (2019). Perspectivas empresariales 2019. Europa y España. Recuperado de https://www.camara.es/sites/default/files/publicaciones/documento_resultados_19_0.pdf

CEOE (2018). Análisis de la empresa exportadora.

Comisión Europea (2018). Proposal for a regulation of the European Parliament and of the Council establishing the InvestEU Programme. Recuperado de https://eur-lex.europa.eu/legal-content/ EN/TXT/?uri=COM\%3A2018\%3A439\%3AFIN

ICEX (2018). La Sostenibilidad como herramienta de internacionalización: nuevas oportunidades para las empresas. Boletín Económico de ICE, (3099).

Instituto de Crédito Oficial (2018). Memoria Anual Integrada 2018. Recuperado de https://www.ico. es/documents/19/1950386/Memoria+Integrada+ 2018

Instituto de Crédito Oficial (2019). Reorientación Estrategia ICO 2019-2021. Recuperado de https://www.ico.es/documents/19/1462960/ PLAN+ESTRATEGICO+CASTELLANO.pdf

Ministerio Industria, Comercio y Turismo (2019). Plan de Internacionalización de la Economía Española 2019-2020. Recuperado de http://www. comercio.gob.es/es-ES/PDF/portada/Plan\%20 Internacionalizaci\%C3\%B3n\%2019-20.pdf

OCDE (2019). FDI in figures. Recuperado de http:// www.oecd.org/investment/FDI-in-FiguresApril-2019.pdf 